\title{
Lapurdum
}

LAPURDUM Euskal ikerketen aldizkaria | Revue d'études basques |

Revista de estudios vascos | Basque studies review

$14 \mid 2010$

Numéro XIV

\section{Pour une anthropologie de la fête au Pays Basque}

\section{Eric Dicharry}

\section{OpenEdition}

Journals

Édition électronique

URL : http://journals.openedition.org/lapurdum/2247

DOI : $10.4000 /$ lapurdum.2247

ISSN : 1965-0655

Éditeur

IKER

Édition imprimée

Date de publication : 1 octobre 2010

Pagination : $33-53$

ISSN : 1273-3830

Référence électronique

Eric Dicharry, «Pour une anthropologie de la fête au Pays Basque », Lapurdum [En ligne], 14 | 2010, mis en ligne le 15 septembre 2014, consulté le 02 mai 2019. URL : http://journals.openedition.org/ lapurdum/2247 ; DOI : 10.4000/lapurdum.2247 


\section{Pour une anthropologie de la fête au Pays Basque}

Eric DICHARRY

«Il y a deux grandes espèces de sceptiques: ceux qui ne croient à rien par paresse, par fatigue, par mollesse, esprits aussi « distingués » que totalement désintéressés de tout. Et ceux qui ne croient que ce qu'ils ont compris, éprouvé, prouvé et vérifié. » (Claude Roy, 1997, p. 235)

«Toute société existe en instituant le monde comme son monde. " (Castoriadis Cornelius, 1975, p. 259)

"La mondialisation, parce que les cultures résistent à l'inculcation, parce que les civilisations sont des structures de longue durée qui canalisent le cours de l'histoire, n'empêche pas chaque groupe social de défendre son identité en recontextualisant les biens importés. " (Warnier, 1999, p.106)

Quel intérêt pour l'anthropologie de s'intéresser à la fête?

Le temps festif est une occasion privilégiée pour saisir les transformations culturelles, politiques, économiques, linguistiques et sociales. La fête est une mise en scène de la société dans son territoire. Elle est définie par les ethnologues comme un temps hors du temps (celui de la quotidienneté). Comme l'écrivait le sociologue Emile Durkheim : «le rythme du temps préside à l'état de congrégation qui apporte à la société le sentiment qu'elle a d'elle-même ». En effet, la fête en scandant le temps apporte à la société le sentiment qu'elle a d'elle-même. Pour Georges Bertin, "L'expression du collectif, dans la fête effervescente, conduit à une épiphanie, une exaltation qui servira de référence dans la grisaille des jours. Elle conforte la résistance à l'imposition sociale». (Bertin, 1999) Ces manifestations collectives (bals, fêtes, banquets, carnavals...) sont des lieux d'initiation. "Ils mettent en oeuvre des rituels par lesquels la société maîtrise le temps qui passe et l'angoisse qu'il suscite. » (Bertin, 2002) La fête permet de maîtriser l'angoisse du temps qui passe.

La fête est paradoxale. Elle est à la fois facteur de cohésion sociale mais aussi rupture, par la transgression de la norme sociale. Elle est «un excès permis, voire ordonné, une violence solennelle d'un interdit » (Freud). Elle permet de saisir écrit Guy Di Meo :

« La façon dont la société construit son rapport au territoire, sa territorialisation et 
ce, notamment dans des espaces sujets à des revendications territoriales. La fête porte une charge identitaire: elle donne du sens à un espace qui devient alors territoire, elle participe ainsi à la territorialisation des lieux en se faisant le vecteur des représentations identitaires. » http://www.cafe-geo.net/article.php3?id_article=111

Il y a incontestablement, en même temps qu'une extension planétaire, une mode et une prolifération de la fête depuis une trentaine d'années et le nombre de recherches qui portent sur cette problématique dans le champ des sciences sociales témoignent de l'intérêt des chercheurs pour cet objet qu'est la fête.

Si elle est tant étudiée, c'est que la fête permet de donner en actes à l'observateur une définition du rapport au monde. Les acteurs de la fête créent un savoir. Un savoir faire la fête : local et collectif. Un savoir sur les gestes, sur leurs corps, sur la convivialité, sur les modes d'interactions, sur eux-mêmes et sur leurs rapports aux autres et au monde. Toute la difficulté pour le chercheur consiste à montrer l'acte de faire la fête en même temps qu'il fait la fête. Pour cet objet d'étude comme pour d'autres, point de salut pour l'ethnologue en dehors du terrain où poussent les faits. La question de la distanciation par rapport à l'objet reste déterminante. Il ne faut pas que sa participation empiète sur sa capacité à observer. Pour ce faire, il doit se positionner tel un être «suréveillé », attentif aux détails qui font sens non seulement pour lui mais aussi et surtout pour ceux qu'il s'est donné comme objectif d'observer. Il s'agit donc de poser en principe une double interrogation. Celle du sens de la fête pour lui-même (observateur) et celle du sens de la fête pour les acteurs (observés). Il se doit donc de problématiser à partir de sa recherche sur la fête 1) son rapport à lui-même, aux autres et au monde et 2) partir de la définition que les acteurs observés donnent en acte de leur rapport à eux-mêmes, aux autres et au monde. La distance se trouve dans la capacité que possède le chercheur à une autoréflexion et à une autocritique permanente qui l'aidera à revisiter cette situation d'acteur et d'auteur, à prendre conscience de son rôle et par là même à valider ses résultats. Comme l'écrit Gérard Althabe:

"Constater que le chercheur est un des acteurs du jeu social dont il s'est donné la tâche de rendre compte, de mettre en évidence la cohérence, de définir les règles, permet de traiter l'enquête elle-même comme un terrain d'investigation; la manière dont l'ethnologue est " produit » en acteur, les transformations dont sa position est le cadre, les relations dans lesquelles il est impliqué font partie de l'univers social étudié et sont élaborées par le mode de communication dont il construit les termes. »(Althabe, 1998, p. 43)

La fête pour revenir à elle, renvoie toujours à une double histoire : personnelle et collective. Mais quand j'énonce que j'aime faire la fête, où quand les acteurs énoncent qu'ils aiment faire la fête qu'est-ce qui se cache sous ces énonciations? Est-ce que la fête renvoie à l'idée de jeunesse? D'ailleurs existe-t-il un âge limite pour faire la fête? Faisons-nous la fête pour rester jeune ou pour se donner à nous-mêmes l'illusion de pouvoir rester jeune? Qu'est-ce que j'aime quand j'énonce aimer faire la fête ? Lactivation mnémonique qui me renvoie aux compagnons avec lesquels j'ai participé aux fêtes? Ce que j'ai été ? Quelles sont mes motivations qui me poussent à faire la fête? Quelles sont les modifications de ces motivations avec les années qui passent? Aimer la fête renvoie-t-il à la vénération de ma propre histoire personnelle? A une histoire qui 
la dépasse en se faisant collective ? Quelles sont les modalités de ma mise en forme et de la mise en forme des autres à travers l'acte de faire la fête?

\section{Un bref détour par l'étymologie}

Etymologiquement, le mot fête provient du latin festi. A Rome, le calendrier était divisé en jours festi, jours de repos consacrés aux dieux et jours fasti, pendant lesquels il était permis de s'adonner aux affaires publiques. Le sens originel de la fête ne se trouve pas dans le simple divertissement mais dans la mise en branle de rites paiens en relation avec les cycles annuels et ses divinités que la population honorait pour rendre propices la chasse, la récolte ou la prospérité de la société. La pratique festive visait à l'origine à calmer et à se protéger des forces de la nature. Elle a gardé avec les fêtes d'aujourd'hui une même préoccupation fonctionnelle quant à la permanence, à la pérennité et à la cohésion de la communauté. Ce n'est que bien plus tard que l'Eglise catholique a superposée ses propres fêtes devenues sacrées sur les dates clés du calendrier solsticien. Fêtes/rites qui comme l'a bien démontré l'historien et anthropologue navarrais Julio Caro Baroja dans les Fête-Dieu espagnoles renvoient à la fois à des croyances anciennes et à des représentations socio-politiques contemporaines.

\section{Le syntagme fête basque en question}

Comme la « culture basque », la " fête basque » n'est pas "la somme algébrique d’un ensemble de traits culturels » (Laborde, 1997). La fête basque ne peut se réduire à une série de termes clairement identifiés. Le syntagme « fête basque » réfère à une double dimension.

"D’une part, il renvoie à des représentations objectales (des objets, des pratiques); d'autre part, il qualifie des représentations mentales, à quoi l'on accède par les discours qui font exister ces pratiques et leur procurent une (ou des) signification(s). Il réfère, en somme, à des opérations sociales dans lesquelles s'opèrent des couplages relationnels associant l'adjectif qualifiant une pratique à un nom qui la désigne. " (Laborde, 1997)

Notre anthropologie de la fête se veut libérée des schémas fixistes et notre théorie de la fête ne découle pas d'un éternel toujours-déjà-là, immuable. Au contraire elle est une manière de qualifier des pratiques festives. Notre analyse de la fête se veut ici résolument dynamique. Il ne s'agit donc pas, pour nous, de simplement définir une fête basque, mais bien d'analyser comment se fabrique, socialement, une fête qui ne devient basque qu'à partir du moment où elle est définie comme telle par les acteurs qui l'inventent. Mais au-delà, ce qui nous importe dans notre recherche, c'est bien plus d'étudier les fêtes que la Fête (cf D. Cuche) Nous aurions donc du intitulé notre conférence " pour une anthropologie de la Fête au pluriel » (cf M. de Certeau) plutôt que «pour une anthropologie de la fête » au singulier.

\section{Entre passé présent et futur}

Dans l'ouvrage Irungo Euskal Jira Federico Abaigar Marticorena évoque l'importance de la récupération d'une part de la culture après la nuit franquiste qui supposa la négation de la différence pour la culture basque. Il revient dans son introduction sur l'importance de la fête 
dans cette reliance entre passé, présent et futur. Il écrit:

"Le passé, source des expériences vécues, n'est pas automatiquement assimilé et il ne se fixe pas de soi-même dans la mémoire collective d'un peuple. L'absence de cette dernière et la carence du savoir accumulé peuvent contribuer puissamment à la faille du passé/présent dans la population d'Irun prononçant une distorsion qui escamoterait la connaissance d'un héritage fort riche. L'histoire se dresserait ainsi en une simple idéologie festive, se vidant de sa sève et nous menant à une atrophie de la mémoire collective d'Irun et de ses habitants. (...) Je ne fais pas référence à quelque chose d'inamovible et d'essentialiste mais à une source dynamique, mobilisatrice des énergies d'Irun, un espoir pour les consciences des gens d'Irun, et en définitive des Basques. (...) C'est le sentiment d'exister en tant que nation, c'est la sensation d'exister avec des désirs d'énergie pour la construction d'un avenir. » (Abaigar Marticorena, 1993, p. 21)

Le discours sur la fête de Federico Abaigar Marticorena est riche d'informations. Il renvoie à la mémoire collective, au savoir accumulé, à l'héritage, au sentiment d'exister en tant que nation, au passé, au présent et à l'avenir. La fête est ici sommée de participer à l'édification et à la construction de la nation basque.

Décrypter et analyser ce discours sur la fête c'est accéder aux significations des acteurs. Significations qui donnent sens aux pratiques festives. La question du prisme de l'analyse de la fête au Pays Basque ou comme nous l'envisagerons plus loin de la fête basque au Pays Basque renvoie quant à elle à celle, plus générique, associée à la culture basque dans son ensemble. Comme l'écrit Denis Laborde:

"Pour tenter de calmer l'effervescence, Jean-Pierre Colin, chargé de mission auprès du Ministre de la Culture pour les cultures régionales et minoritaires, est aussitôt dépêché sur place. Il vient rencontrer les acteurs du Centre Culturel, à Bayonne, les 7 et 8 juin 1985. Il affiche, dans le journal Sud-Ouest du 8 juin, la volonté du gouvernement de "défendre le basque au titre des cultures du monde ". Là encore, dans le discours du fonctionnaire ministériel, l'action en faveur d'une culture basque se prononce sur le mode de la défense, c'est-à-dire sur le mode d'un ralliement à la cause des offensés et des humiliés (un misérabilisme). Cette attitude coïncide, comme "nécessairement ", avec un rejet au loin d'une culture basque comme l'une, parmi bien d'autres, des "cultures du monde ». La conjugaison de ce misérabilisme et de ce procès en décontextualisation fait de la "culture basque " une pure abstraction. L’on ne quitte pas ici le registre polémologique qui était déjà celui des folkloristes du XIX siècle. Pour eux, un « savoir du peuple " (folklore) ne méritait d'être étudié qu'à la condition de le rejeter au loin sur un axe historique, c'est-à-dire de n’y voir qu'un ensemble de survivances d'un état antérieur de la civilisation. " (Laborde, Lapurdum, 1997)

Lanalyse qui porte son regard au loin sur un axe historique ne mérite d'être mentionnée qu'à la condition qu'elle puisse renseigner le chercheur sur la dimension dynamique du rite et/ ou des fêtes qu'il se propose d'étudier. Plutôt que de nous intéresser à la fête basque comme une pure abstraction qui ne mérite d'être étudiée qu'à la condition d'être rejetée au loin sur 
l'axe historique, nous nous intéressons à la réalité de l'acte et aux sens que les acteurs donnent à ces actes. Interroger anthropologiquement la fête c'est appréhender la fête de manière à la fois diachronique et dynamique pour pouvoir penser les modifications qui l'affectent et en retour pouvoir analyser les transformations économiques, sociales, linguistiques, démographiques, politiques de la communauté qui lui permet de prendre corps, de s'inventer et de sans cesse se réinventer. En ce sens, la fête permet une lecture anthropologique de la communauté qui l'invente. Mais, en retour, la fête réinvente sans cesse la communauté. En d'autres termes, la communauté réinvente en permanence la fête qui à son tour la réinvente. Pour prendre un exemple puisé au Pays Basque Nord, nous pourrions développer notre idée en disant que les mascarades tout comme les pastorales inventent un territoire mais aussi, et surtout, une population qui y réside. La Soule ne serait plus tout à fait la Soule sans ses mascarades et ses pastorales. Il faudrait alors interroger les modalités de ces inventions en analysant selon quelles modalités les mascarades et les pastorales inventent les Souletins qui les inventent.

Fête et sens des acteurs

Pour l'anthropologue, la fête, qui s'inscrit toujours dans un espace et dans un lieu, renvoie à une collectivité qui se met en exergue à travers des pratiques rituelles. Interroger anthropologiquement la fête c'est interroger les modalités du sens que les acteurs participants aux rites festifs accordent à ces rites. C'est comprendre l'importance qu'ils leur accordent. Comme l'écrit Xabier Itzaina :

"Le linguiste L. Michelena soulignait que le mystère de la langue basque ne venait pas tant de sa nature que de sa pérennité. De même, la particularité de la Fête-Dieu du Pays Basque ne vient-elle pas de ses caractéristiques, mais plutôt de sa permanence. Le "stock " de gestes, de musiques, de costumes et de pas exhibés à cette occasion évoquent en grande partie un modèle festif très répandu dans l'Europe pré-révolutionnaire. La permanence des formes, cependant, génère de plus en plus d'interrogations autour de leur signification. Or, il s'agit de distinguer la signification intrinsèque prêtée au symbole et le sens du même signe tel qu’il est visé par les acteurs du rituel. Les deux dimensions s'imbriquent pour constituer la réalité de l'acte. Il est en ce sens abusif de rejeter comme fausse l'interprétation très répandue dans les villages de labourdins et bas-navarrais: les costumes et la musique seraient des legs de l'époque napoléonienne, ramenés par quelque grognard de passage, etc. Le problème n'est pas tant de savoir si les soldats de l'Empire se sont ou non arrêtés à Iholdy, mais plutôt de comprendre pourquoi les gens d'Tholdy ont construit et maintenu cette explication. La Fête-Dieu, en ce sens, attend toujours son herméneutique. Les représentations des acteurs dévoilent des croyances et des valeurs, en un mot, sont des révélateurs de sens. » (Itzaina)

Fête et identité entretiennent des relations intimes. Pitxu est l'emblème de la Soule voire du Pays basque au même titre que les pastorales. Une Soule sans pastorales ne serait plus tout à fait la Soule. Et c'est précisément parce que les mascarades tout comme les pastorales participent à la définition de ce qu'est la Soule pour les Souletins et les Basques dans leur ensemble qu'elles perdurent. C'est précisément parce que la fête fait sens et participe à la définition de l'identité de ceux qui l'inventent qu'elle perdure. Et apparemment au vu de la pérennité des pastorales et des mascarades il est possible d'affirmer que ces fêtes continuent de 
jouer un rôle dans la définition que les Souletins se donnent d'eux-mêmes à eux-mêmes et au reste du monde. Tant que les Souletins continueront de se définir comme des habitants de la province de Soule où se déroulent des pastorales et des mascarades, ces rites perdureront.

Mais cette pérennité peut parfois poser des questions qui révèlent des paradoxes. En effet, pour monter des mascarades, il est nécessaire que le village qui les monte possède non seulement des acteurs, des danseurs et des chanteurs mais également des locuteurs bascophones. Or, avec la crise linguistique que connaît la province, de moins en moins d'habitants maîtrisent la langue basque dans sa version locale souletine. Pourtant les Souletins ne sont toujours pas prêts en ce début de XXIème siècle à monter des mascarades où les textes des acteurs se donneraient à entendre en français. Ils ne sont pas non plus prêts à réintégrer le drapeau français dans la troupe. Une fois encore, ce refus de monter des mascarades en français avec des symboles français participe d'une définition que les Souletins tentent de se donner d'eux-mêmes à eux-mêmes et au monde. En montant des mascarades ils tentent de perpétuer une identité à laquelle ils se raccrochent car elle les continue de les constituer dans leur être. Dépossédés de mascarades, ils se sentiraient en quelque sorte dépossédés d'euxmêmes, comme dépossédés de leur être. Dépossédé de ce qui les constitue dans ce qu'ils sont, les Souletins ne pourraient dès lors plus se définir comme Souletins face aux Labourdins, aux Bas-navarrais, aux Biscayens, aux Guipuzcoans, aux Basques du Sud, aux Espagnols ou aux Français. Face à cette possible hypothétique dépossession ils préfèrent se projeter dans une Soule basque car bascophone. Et c'est ce possible ancrage dans cette basquité que proposent les mascarades et les pastorales. C'est précisément en scandant le temps à coups de mascarades et de pastorales qu'ils se donnent le « sentiment » d'y parvenir. Les mascarades et les pastorales sont en quelque sorte comme la partie visible de l'iceberg, comme l'arbre qui cache la forêt, un « spectacle » qui donne la possibilité aux Souletins de se donner un visage dont ils se sont euxmêmes privés aidés en cela par un contexte diglossique défavorable. Paradoxalement à mesure que la langue quittait les locuteurs les signes de "Soulinité » se faisaient plus efficients. Les anthropologues auraient donc tout intérêt de se méfier des « signes extérieurs d'identité » pour se pencher sur les raisons qui président à ces mises en représentations spectaculaires.

Nous n'adhérons pas à l'analyse de Guy Di Meo de l'Université de Pau et des Pays de l'Adour selon laquelle la fête est subversive en apparence. Certes, elle remplit une fonction spatiale régulatrice, puissamment identitaire mais elle peut également conserver sa fonction contestataire profondément anti-conservatrice. Les mascarades souletines sont un bon contre exemple pour permettre de comprendre que la fête, incarnée en Soule par son carnaval, en tentant d'imposer un nouvel ordre social, politique et linguistique qui s'étalerait sur un temps bien plus long que celui strictement festif propose par le truchement de l'idéologie abertzale un futur correspondant à ses attentes. Une nouvelle société basque "rebasquisée » distincte de celle qu'elle est dans la réalité. En ce sens, les auteurs des textes ne se contentent pas de faire « dire pour dire » les acteurs. Les énoncés insistent sur l'importance de « faire faire » leurs allocutaires. Ils les exhortent à passer à l'action, à réinventer une Soule bascophone ancrée sur ses traditions. Le dire et le dit pensés pour « faire faire » ne peuvent donc pas simplement être pensés pour conforter la société dans ce qu'elle est mais bien pour la pousser au changement. Plus que de fonction conservatrice du rite, il faudrait donc bien dans ce cadre parler d'une fonction régénérative, d'une fécondation par utopie en marche interposée, d'une dynamique 
à insuffler. Les mascarades installent le conflit pour pouvoir faire en sorte que la population se saisisse de son dépassement. Mais ce dépassement n'est jamais totalement pensable, du moins sur le court terme, puisque précisément il est de l'ordre de l'utopique. Tant que la structure sociale et le contexte linguistique correspondait aux attentes des acteurs, la fonction conservatrice de la fête était respectée. Le sens de la fête était bien dialectique. Les mascarades installaient le conflit pour pouvoir mieux le dépasser. Mais dès lors que la réalité sociale, identitaire, politique et linguistique était devenue problématique aux yeux des acteurs, elle ne pouvait plus être tolérée. Les mascarades devaient donc être mises à contribution en vue d'un changement et servir à une prise de conscience collective. Les mascarades ne possèdent donc pas en elles-mêmes les clefs du dépassement des « conflits » qu'elles initient. Au contraire, elles sont utilisées par les producteurs de discours partisans du changement à des fins contestataires et anti-conservatrices et ce pour faire en sorte que les conflits initiés puissent s'émanciper hors du cadre stricto sensu de la pratique culturelle.

La «non fête » est tout aussi prolixe en enseignement. Larrêt définitif ou l'interruption momentanée sont également à interroger car ils permettent d'analyser les raisons d'un désamour pour un rite, pour une fête, pour une danse ou encore pour des chants qui, s'ils disparaissent, ont toujours de bonnes raisons pour le faire. Il s'agira alors d'interroger les fonctions de la fête pour savoir si elles sont toujours opérationnelles. Lobjectif sera de rechercher dans le discours que les acteurs tiennent sur la fête, les raisons d'une reprise ou d'une non reprise après plusieurs années voire décennies d'interruption. Une bonne manière d'aborder la question consisterait à interroger les acteurs sur les raisons de l'obsolescence de tel ou tel rite, de telle ou telle danse ou de tel ou tel instrument. Le txistu par exemple peut être le point de départ d'une analyse anthropologique. Comme l'écrit Federico Abaigar Marticorena:

"Le son du txistu et du Tambourin qui a toujours convié la jeunesse à danser, commence à devenir obsolète, se bornant aux fêtes champêtres, aux exhibitions de danse traditionnelle, et autres évènements particuliers. Aujourd'hui, la majeure partie de notre jeunesse préfère la musique disco, le heavy metal y compris les rancheras et les pasodobles. Tout cela avec le plus grand nombre de watts qui puissent s'y concentrer. " (Abaigar Marticorena, 1993, p. 245)

La description puis l'interprétation de l'évolution des usages du txistu dans les fêtes au Pays Basque par une recherche menée sur le terrain facilitée par une observation participante voire par une " participation observante " (Dicharry, thèse, 2009) serait riche d'enseignements. Il ne s'agira pas dans ce cadre pour l'anthropologue de porter un jugement sur cette évolution mais bien plus d'en rendre compte de la manière la plus objective possible en interrogeant le discours que les acteurs qui participent aux fêtes tiennent sur le txistu. Interroger le pourquoi de cette préférence pour la musique disco au dépend du txistu comme le laisse entendre Federico Abaigar Marticorena serait sans nul doute particulièrement enrichissant. Comme il serait tout aussi intéressant d'interroger par exemple les raisons pour lesquelles un tel auteur ressent une vive émotion lorsqu'il évoque les chemises de lin portées pendant les fêtes d'Irun à la fin du XXème siècle qui sont : « de la même qualité que celle de ses ancêtres » (idem, p.248). Questionner « la recherche d'authenticité » serait aussi riche d'enseignement. 
Ne pourrait-on pas en effet envisager de repérer un jour dans une fête un joueur de txistu pratiquant son instrument habillé avec un t-shirt reprenant le logo ou le visuel de son groupe préféré de heavy metal basque ou non basque? Aimer le heavy metal et pratiquer le txistu ne sont pas en effet incompatible! En quoi ce txistulari serait-il alors moins appréciable qu'un autre txistulari jouant avec une chemise en lin ? Ne vaudrait-il d'ailleurs pas voir un txistulari de ce type plutôt que de ne pas voir du tout de txistulari ? Un tel txistulari permettrait sans doute d'attirer à lui toute une série de jeunes qui verraient dans cette pratique une pratique vivante car capable de se réinventer sans cesse. Car nous le savons bien, une culture qui cesse de se réinventer est une culture qui se meurt. Pour prendre un autre exemple tiré du monde bertsularistique nous pourrions dire que ce n'est pas parce que les jeunes improvisateurs basques ont laissé de côté leurs bérets qu'ils ont cessé d'être des improvisateurs basques. Ce qui est d'ailleurs particulièrement frappant, c'est d'observer ces jeunes habillés au goût du jour mais qui dans leurs pratiques ont su garder des règles de composition formelles et des airs traditionnels comme autant de signes d'une transmission réussie et opérationnelle. Opérationnelle car la culture transmise fait sens pour eux. Ce "faire sens » en lien avec le contenu des bertsus sans cesse réinventés dans l'instant étant la condition sine qua non pour une possible réinvention.

Faire la fête oui mais comment?

Il existe bien en réalité une diversité de fêtes. En effet quoi de commun entre une fête tekno qui a lieu un samedi soir dans une discothèque au Pays Basque sud et où participent des jeunes de la côte basque originaires de Biarritz et de Bidart et une fête souletine comme par exemple les mascarades qui se déroule selon des cadres prescrits par la tradition (organisation des journées, danses, textes, costumes, chants, repas, déroulement, calendrier...) et où participent des Souletins mais aussi des habitants du Pays Basque sud? Très peu de choses si ce n'est une même envie de participer à des réjouissances, un même désir revendiqué par les acteurs de faire la fête.

Sous cette expression « faire la fête » se dessinent une multiplicité de pratiques, d'attitudes, de comportements et de manières de faire qui sont parfois difficiles à rapprocher les uns des autres. Derrière le terme générique de fête se cachent une multitude de déclinaisons. Sous un même désir de "faire la fête " bien des motivations apparaissent. Motivations qui renvoient à autant de fonctions «personnelles » qui nourrissent les discours des acteurs sur la fête : fuir la réalité, retrouver des proches, passer du bon temps, se libérer, s'amuser, s'enivrer, décompresser, revendiquer, critiquer. Mais à ces fonctions « individuelles » viennent s'ajouter des fonctions essentielles pour la communauté. La fête favorise en effet la cohésion et l'homogénéité du corps social. Elle renforce symboliquement le sentiment d'appartenance à un groupe. Elle a une fonction de conservation. Elle renouvelle périodiquement et de manière cyclique les croyances du groupe, permet de relier le présent au passé et inscrit les membres de la communauté dans une histoire qui les dépasse en tant qu'individus. Elle a une fonction transgressive par renversement de l'ordre communautaire par l'ordre festif. Renversement qui légitime l'intégration de revendications et de critiques (politiques, sociales, linguistiques, économiques...). Critiques et revendications d'autant plus légitimées qu'elle font partie de l'essence même du rite festif. Elle a enfin une fonction régénératrice et purificatrice. Elle 
permet à la société et aux membres qui la composent de se libérer de leurs pulsions. La fête est donc un excès autorisé, toléré sous certaines conditions et dans certaines limites. Toutes les fêtes ont en commun de proposer à ceux qui les vivent un moment de rupture avec la vie quotidienne. Rupture temporelle : les fêtes s'inscrivent en rupture avec le temps de la vie de tous les jours. Elles sont à la fois commémoration par réitération et abolition du temps. Rupture de l'espace : la fête délimite la communauté (intérieur / extérieur) et lui permet d'intégrer de nouveaux membres (rites d'initiations), elle favorise les passerelles entre monde réel et imaginaire (carnaval, bertsularisme) entre monde des vivants et des morts (résurrection de Pitxu). Elle crée un nouveau monde (inversion carnavalesque, vérité bertsularistique).

Les deux exemples évoqués plus haut (fête tekno et mascarades) suffisent à mettre en exergue la «polysémie », la pluralité des possibles liés au concept de fête. Sous ce terme le chercheur pourrait classifier toute une série de fêtes et de pratiques festives. C'est pour préciser ce que nous entendons par « anthropologie de la fête au Pays Basque » que nous avons décidé aujourd'hui de prendre la parole car il existe un monde entre le fait d'aller gober des pastilles d'extasy dans une discothèque et le fait de tenir une buvette pour récupérer des fonds pour un parti politique. Un monde qui, illustré par ces exemples, permet de mettre en exergue une multitude de manières de faire la fête au Pays Basque.

Pour une classification : spatiale et temporelle

Il existerait donc fêtes et fêtes. Comment dès lors les classifier. D'une inscription dans le temps et dans l'espace découlerait une première classification de fêtes : fêtes de villes, fêtes de villages, fête de province, fête d'un soir, fête de fin de semaine, fêtes liées au calendrier religieux (Noël, Pâques...), fêtes païennes, fêtes traditionnelles, fêtes locales, nationales, internationales. A cette classification spacio-temporelle, nous pourrions inclure d'autres éléments classificatoires comme par exemple l'âge des participants. La fête tekno évoqué en début de conférence rentrerait dans la rubrique «fête de jeunes». Fête de jeunes qui aurait son équivalence avec des fêtes où ne participent que des personnes âgées. Car même si la jeunesse et la fête restent intimement liées l'une à l'autre il ne faudrait pas croire que seuls les jeunes font la fête. Lactivité des jeunes viendrait parfaire la classification. L'exemple des fêtes étudiantes illustrerait notre propos. A ces fêtes inscrites dans un temps et un territoire viendraient s'ajouter les fêtes ayant un rapport avec un produit : fête du cidre (sagarno eguna), fête du jambon, fête du chocolat (Bayonne par exemple fête son chocolat, chaque année, lors du week-end de l'ascension), fête du gâteau basque (organisée à Cambo). A ces « fêtes produits » viendraient s'ajouter d'autres fêtes devenues tout aussi commerciales : fête des amoureux (Saint Valentin), des mères, des pères, des grands-mères, des grands-pères. Et la liste des fêtes serait incomplète si nous n'évoquions pas la fête de la nature, de la musique, de la poésie (printemps des poètes), du cinéma, du goût, du livre, et même la fête du travail. Une autre classification celle là d'ordre « culturel » viendrait clore ce chapitre classificatoire. Il existerait bien des fêtes basques voire des fêtes de Basques. Nous reviendrons au cours de cette prise de parole sur cette notion de « fête basque ». Il y aurait au Pays Basque toute une série de fêtes et certaines bien qu'elles aient lieu sur le territoire basque ne mériteraient pas au dire de certains le qualificatif de basques. Nous aurons dès lors à nous interroger sur ce qui constitue au dire de certains Basques les fondations de cette «basquité » de la fête basque au Pays Basque. 
Mais pour l'heure, restons à Bayonne pour évoquer ses fêtes. Pour élaborer cette conférence, j'ai tout d'abord été surfer sur la toile en tapotant sur mon clavier fête au Pays Basque. Je n'avais pas terminé de rédiger l'intégralité de mon texte que des liens apparaissaient avec simplement le mot «fête ». Et c'est aux fêtes de Bayonne que m'a immédiatement renvoyé ce mot fête. Le premier site s'intitulait : " fête de Bayonne site officiel ». J'ai cliqué sur le lien et toute une série d'information apparurent. La date des fêtes, du 27 au 31 juillet 2011, le programme, le plan, la présentation et un compte à rebours. Puis trois autres rubriques intitulées : «l'indispensable», «pour que la fête soit belle» et « actualités ». Dans la première rubrique intitulée «indispensable», je pouvais trouver les informations pratiques (plan, transport...). Je téléchargeais un document en PDF et pouvais lire : porter la tenue des fêtes en blanc et rouge n'est pas le seul signe de reconnaissance du festayre. Porter un badge ce serait s'engager à porter la bonne parole, à respecter la charte du festayre, celle du bon « savoir fête », ce serait contribuer à ce que la fête se déroule dans les meilleures conditions.

Selon ce site internet dédié aux fêtes de Bayonne il existerait donc un "savoir fête » renvoyant à un « savoir faire la fête » qui serait non seulement fonction d'un endossement de l'habit traditionnel festif en rouge et blanc mais aussi de toute une série d'attitudes, de manière de faire, de comportements à avoir ou à proscrire. Ces manières de faire renverraient à une « culture de la fête », c'est-à-dire à des manières de sentir, de penser et d'agir en période de fête. Culture de la fête transmise localement et oralement de générations en générations ou qui par défaut se devait d'être transmise par le biais d'un site internet à vocation « pédagogique ».

Toutes ces informations nous sont précieuses à l'heure de définir ce qu'est la fête. C'est d'abord un temps, une période, avec une date de début et de fin. Un temps réitéré d'années en années. Un temps cyclique qui fournit à la fête son inscription dans l'histoire. Fête " temporalisable », répérable dans le temps, qui peut permettre aux chercheurs d'écrire son histoire, d'analyser son évolution, ses transformations. En surfant sur la toile le chercheur constate alors que les fêtes de Bayonne possèdent même un article sur l'encyclopédie libre Wikipedia où il est écrit :

"Les fêtes de Bayonne sont une période de fête dans la ville basco-gasconne de Bayonne (Pyrénées-Atlantiques) qui débute le mercredi qui précède le premier week-end du mois d'août et se termine le dimanche suivant (en cas de week-end à cheval sur les deux mois, elles s'achèveront toujours le premier dimanche d'août). En 2004, ces fêtes réunissaient entre 1,3 et 1,5 million de visiteurs, faisant de ces fêtes les plus importantes de France. La tenue de rigueur est blanche, accompagnée d'un foulard et d'une ceinture rouge (cinta ou faja). Cette tenue, non traditionnelle de la région, provient en fait de la Navarre en Espagne. La ville est membre du l'Union des villes taurines françaises. Durant les fêtes, deux corridas ont lieu. "

Nous retrouvons dans cet article les éléments définitoire de la fête. Son inscription dans le temps mais aussi et c'est là le second élément qui entre en ligne de compte dans la définition de la fête son inscription dans l'espace. Les fêtes sont localisables, attachées à un lieu, à un espace, à un territoire. Les fêtes de Bayonne ont lieu à Bayonne, de telle date à telle date et nulle part ailleurs. C'est ce qui explique son originalité, sa singularité et sa non exportabilité. C'est ce qui 
explique parfois son succès, sa renommée et son attractivité (1,3 et 1,5 million de visiteurs), qui renvoient tous au hic et nunc, à l'ici et au maintenant. Inscription spatio-temporelle sur laquelle viennent s'inscrire des manières de faire la fête, de s'habiller avec une tenue de rigueur aux couleurs fixées par la tradition mais aussi des évènements factuels. La fête c'est le moment nous informe Wikipedia où tels et tels évènements ont lieu. Ici c'est le temps des deux corridas mais aussi le temps des parties de pelote, des concerts, des bals et des danses sur les places, des courses de vachettes, du corso lumineux, des défilés de chars et de bandas, des toros de fuego et des feux d'artifice qui sont autant de moments forts qui animent les fêtes. Chaque animation s'inscrit dans un espace propre. Les danses basques muxiko dantza par exemple sont réalisées sur la place du château vieux. Le tournoi de pelote à main nues est organisé au trinquet moderne. Chaque fête possède ses rites. A Bayonne c'est le rite d'ouverture avec la « marionnette » du roi Léon qui lance depuis l'année 1947 les trois clés des trois quartiers de la ville (Grand Bayonne, Petit Bayonne et Saint Esprit) du haut du balcon de la mairie avec une personnalité invitée et un bertsulari qui chante un bertsu. Trois messes symbolisant les différents quartiers de Bayonne sont célébrées le dimanche avant la clôture.

Chaque fête a son histoire propre, ses singularités mais elle possède aussi avec les autres fêtes des dénominateurs communs. Côté vestimentaire, celles de Bayonne a connu des modifications. Lors des premières fêtes, en 1932, les couleurs étaient le bleu et le blanc. Le bleu venait du bleu de travail des ouvriers. Ce bleu fut ensuite remplacé par le rouge, à l'instar des fêtes de Pampelune où le rouge et le blanc sont les couleurs officielles. Une polémique continue de nos jours, une minorité préférant les bleu et blanc originels. Couleurs qui peuvent se rapporter aussi aux couleurs du club omnisports le plus important de la ville, l'Aviron bayonnais.

La fête est donc un moment spécifique, à part dans la vie de la communauté. Un moment de célébration et de réjouissances publiques qui se distingue de la vie de tous les jours. Le temps festif s'inscrit en rupture avec le reste des jours de l'année. Comme le dit une phrase du langage commun : «ce n'est pas tous les jours fête ». La fête est un moment délimité dans le calendrier de la cité où les participants à la fête peuvent faire parfois ce qu'ils ne feraient pas d'habitude. Un moment extraordinaire propice aux transgressions d'interdits et aux amusements licencieux. La fête renvoie aux limites entre légalité et illégalité, entre licite et illicite. Et en la matière il y a des limites à ne pas dépasser. Comme l'écrit Isabelle Garat :

«(...) les débordements ne sont autorisés que dans un certain seuil sous le regard de la police, des municipalités, des services de santé lesquels soumettent à autorisation les rassemblements dans l'espace public, les transgressions ont de moins en moins de sens, ainsi en est-il de la transgression de l'identité sexuelle (hommes enceintes, hommes déguisés en femmes), le gaspillage perd de son sens dans une société de consommation, ou il le renouvelle, de l'excès de table de la ripaille carnavalesque, on passe à l'alcoolisation de plus en plus jeune et plus excessive qu'autrefois, ou à la multiplication des événements consommatoires (fêtes des grands mères, festivals et fêtes à foison). » (Garat, Isabelle, 2000)

La collectivité reconnait et concède à ceux qui font la fête une certaine liberté, certes, mais cette liberté est somme toute relative. Elle est encadrée. Les autorités imposent de respecter 
des horaires de fermeture de bars et de peña. Chaque jour, il existe une " heure légale de fermeture des fêtes ». Il y a des bornes à ne pas dépasser et ces bornes se réfèrent à la morale et à la législation. Si l'ivresse sur la voie publique est tolérée voire largement acceptée par temps de fêtes, enfreindre certaines autres lois ne fait pas partie des limites autorisées. La collectivité s'autorise même la possibilité de rappeler à la loi et de juger ceux qui auraient dépassés ces limites. C'est le cas par exemple lorsque le parquet est saisi pour des consommations et des trafics de produits stupéfiants, des infractions au code de la route, des conduites en état d'ivresse, des viols, des viols en réunion, des coups et blessures volontaires, des bagarres, des rixes et des violences diverses et variées.

\section{Fête et identité}

Fête et excès en tous genres sont souvent synonymes. Lactivité des postes de secours lors des fêtes de Bayonne est là pour rappeler que les conséquences des nuits d'ivresse et de liesse peuvent être parfois, pour certains traumatisés, irrémédiables. Mais pourtant, malgré ces débordements et ces excès, les fêtes reviennent d'année en année. Qu'est-ce qui explique alors cette inscription des fêtes dans le temps malgré ces quelques graves incidents relayés chaque année par la presse locale et nationale ? C'est du côté des fonctions remplies par la fête qu'il faudrait rechercher son inscription dans le temps. La fête constitue en premier lieu un marqueur d'identité. Bayonne ne serait plus véritablement Bayonne si on la privé de ses fêtes. Car Bayonne, aux yeux des Bayonnais mais aussi aux yeux d'une population exogène à Bayonne est définie dans ce qu'elle est par les fêtes qui s'y déroulent chaque année depuis maintenant près de 70 ans. Priver Bayonne de ses fêtes ce serait alors privé Bayonne d'une partie de ce qu'elle est. Ce serait en quelque sorte la « dénaturer ». C'est ce qui explique la difficulté pour tout politique de venir interférer et/ou légiférer sur la fête. Toute réglementation de la fête entraînant aussi tôt une levée de bouclier qui ne peut s'expliquer uniquement par les retombées économiques de la fête. Et ce qui est vrai de Bayonne l'est tout autant pour d'autres villes de part le Pays Basque et le monde. Il nous serait en effet bien difficile d'imaginer Rio et Venise sans leur carnaval, Munich sans sa fête de la bière où Pampelune sans ses san fermin. La fête renvoie au concept d'identité et ce concept est particulièrement important et opérant pour tous ceux qui souhaitent réaliser une anthropologie de la fête. La fête est ce qui défini un espace, un territoire, une ville, un village, une province, un pays mais aussi une population dans son identité.

Les Basques ont largement été définis de la sorte par de nombreux auteurs, comme un peuple qui aime danser et chanter c'est-à-dire comme un peuple qui aime faire la fête. Mais cette définition des Basques est avant tout interne, endogène. Elle n'est pas uniquement le fait d'une définition exogène aux Basques. L'exemple de la danse et du chant en donne une parfaite illustration. Ces deux pratiques font partie avec la langue des marqueurs de l'identité basque c'est-à-dire qu'ils participent à la définition que les Basques se donnent d'eux-mêmes. S'il nous fallait alors repérer une basquité dans les fêtes de Bayonne c'est du côté de ces pratiques culturelles considérées par les Basques eux-mêmes comme faisant partie de ce qui les définit en tant que Basque qu'il faudrait nous attarder. Pour retrouver une fête basque au Pays Basque, et plus spécifiquement à Bayonne pendant ses fêtes, c'est donc du côté des moments de danses et de chant qu'il faudrait mener une investigation anthropologique. 
Ce serait par exemple aussi, dans un autre espace-temps, interroger la place des bertsularis dans les rites d'ouvertures des fêtes, en retracer l'histoire, analyser les productions, mener des entretiens avec ces improvisateurs pour connaitre le sens qu'ils donnent à ces interventions. Ce serait également partager, avec des "fêtards », des moments festifs dans des espaces où les bascophones font la fête en basque et repérer en quoi ces manières de faire la fête ou ces « savoirs fêtes » diffèrent des « savoirs fêtes » des fêtards non bascophones.

Lanthropologue pourrait alors repérer des espaces propices à l'accomplissement d'une fête basque singulière (des rues, des bars, des places,...) et déceler (derrière des pratiques festives originales et singulières qu'il lui incomberait de décrire, d'analyser et d'interpréter), des discours qui les soutiennent et les motivent. Des discours emprunts de tonalités revendicatives d'ordre linguistique voire politique. Lanthropologue pourrait à partir de ces discours festifs politiquement et linguistiquement colorés déduire entre autre que la revendication politicolinguistique est la signature de la singularité de la fête des Basques au Pays Basque. La rédaction d'une recherche sur la question lui permettrait de retrouver sous la plume d'auteurs bascophones une confirmation de ses hypothèses. Pour ceux bascophones qui sont intéressés par la question abordée ici nous conseillerons vivement la lecture de l'ouvrage d'Eneko Bidegain intitulé: «Patxa. Besta bai borroka ere bai » paru aux éditions Gatuzain.

Un rapide survol anthropologique de la fête au Pays Basque permettrait d'élaborer une distinction entre d'un côté « la fête au Pays Basque » et de l'autre « la fête basque au Pays Basque ». Car comme nous avons pu le présenter brièvement, toutes les fêtes au Pays Basque sont loin de pouvoir mériter le qualificatif de basque car pour qu'une fête puisse endosser le qualificatif de basque il ne suffit pas, au dire des acteurs, qu'elle se pratique sur le sol basque. Il lui faut au dire de certains Basques autre chose. Attardons nous maintenant sur cet « autre chose », sur ce " plus », qui légitimerait à lui seul cette possible qualification. Pour définir l'objet d'une anthropologie de la fête basque au Pays Basque, le chercheur devrait focaliser son attention sur les fêtes revendiquées comme basques par les Basques eux-mêmes. A ce niveau de la recherche, il serait d'ailleurs intéressant d'analyser les distinctions apportées par les Basques à ces affirmations de basquités car il existerait bien un monde entre " fêtes basques » et « fêtes basques». Quoi de comparable en effet entre un spectacle estival folklorique pour touristes en mal d'authenticité et une fête en basque destiné à des Basques et revendiquée comme basque par les acteurs de cette fête. Le discours de Basques qui critiquent la production culturelle d'autres Basques est à ce titre révélateur de distinctions et de positions qualifiantes et disqualifiantes.

Un détour par la joute organisée en faveur de la radio en langue basque Gure Irratia à l'Atabal de Biarritz le vendredi 25 septembre 2009 va nous permettre d'éclairer notre propos. Joana Itzaina, la meneuse de jeu, en langue basque gai emailea, met entre les mains du bertsulari Sustrai Colina un compact disque. Sustrai Colina doit poursuivre son improvisation à partir de ce disque. Sustrai regarde le disque, il le tourne et le retourne puis le montre au public qui commence à rire. C'est un album de la chanteuse Anne Etchegoyen. Si le public rit c'est qu'il connait les « goûts musicaux» de Sustrai Colina et ce genre de musique n'en fait pas partie. Lassistance s'attend donc naturellement à ce que l'improvisateur fasse une critique en bonne et due forme de la production de l'artiste. C'est ce qu'il ne manque pas de faire. 


\section{Sustrai Colina}

Jakin zazute nere CD hau / Anne Etxegoien dala ta Patxamama jartzen du hemen / sinistu ezin den bezala. Norbaitek ber(e)hau nahiko baluke / etorri 'ta har dezala neretzat ez da produktu on bat / ez da ere kulturala. Baizik paristar handi usteei / saltzen dieten postala

(Sachez que mon CD est celui d'Anne Etchegoyen / Et il est inscrit sur la couverture un Patxamama qui n'est pas crédible / Si quelqu'un veut le faire sien qu'il vienne et qu'il le prenne / A mon avis ce n'est pas un bon produit ce n'est même pas de la culture / Seulement une carte postale destinée à être vendue aux parisiens )

Sustrai Colina fustige, honnit, désapprouve et éreinte le travail de la chanteuse. Il critique le fait qu'elle se soit permis de choisir comme titre de son album Patxamama. Il faut comprendre ici que pour Sustrai Colina ce choix est une tentative de récupération d'un concept à des fins commerciales. Il considère enfin que ce CD n'est même pas culturel (kulturala), en d'autres termes que ce n'est pas de la culture, mais qu'il est simplement destiné à conforter les Parisiens dans l'image folklorique « carte postale » stéréotypée qu'ils se font du Pays Basque. Un beau pays touristique où les gens chantent et dansent. Son Pays Basque à lui est différent de cette image glamour véhiculée par les mass médias et certains artistes basques, un pays qui lutte pour son autodétermination, un pays qui subit de plein fouet les attaques répressives des forces de répression (Garde civile, justice partiale de Madrid, services secrets espagnols, police et gendarmerie française, renseignements généraux) qui édictent et appliquent des lois oppressives qui ne respectent pas les droits des Basques. Un pays où plus de 700 de ses habitants pourrissent dans les geôles et doivent subir quotidiennement des traitements dégradants. Lon assiste donc à la mise en opposition de deux mondes basques. L'un à l'image d'une carte postale (postala) destiné aux touristes et proposé par l'artiste Anne Etchegoyen, l'autre en lutte d'un Sustrai Colina. Il propose une culture dans le sens Todorovien du terme : « une classification du monde qui nous permet de nous orienter plus facilement; c'est la mémoire du passé propre à une communauté, ce qui implique aussi un code de comportement dans le présent, voire un ensemble de stratégies pour l'avenir » (Todorov, 1989, p. 337). Les applaudissements du public laisse envisager que sa préférence est entièrement acquise à ce second monde.

Dans l'exemple ci-dessus, l'auditeur Sustrai Colina dans sa rhétorique basquisante n'identifie le $\mathrm{CD}$ de la chanteuse Anne Etchegoyen ni à de la culture, ni à fortiori à de la culture basque. Au contraire il le disqualifie en affirmant que ce n'est pas un bon produit. En disqualifiant cette production, il érige en lieu et place une autre culture, la sienne qu'il partage avec son public devenu son complice par connivence. La vraie culture, le « vrai » chant basque, la véritable fête basque de la langue basque, est incarnée par le bertsularisme c'est-à-dire par une pratique culturelle réalisée en langue basque, par des Basques, pour des Basques. Pratique reconnue à la fois par les improvisateurs et par leur public comme une pratique culturelle identifiée comme basque. En ce sens, pour reprendre à notre compte une posture interactionniste, la fête basque existe bien, à un moment donné et dans un contexte mouvant où, dans un face à face, un fêtard fait la fête avec d'autres fêtards qui reconnaissent 
cette fête et qui l'identifient comme basque.

Mais cette notion de fête basque mérite d'être explicitée, creusée, disséquée. Existerait-il une «vraie » et une «fausse» fête basque comme il existerait une «vraie » et une «fausse » culture basque, un vrai et un faux chant basque? Une « vraie fête basque » revendiquée comme telle par certains Basques et constituée de pratiques qualifiées comme réellement basques (bertsularisme, théâtre en langue basque, chant pratiqué par des chanteurs qui parlent et comprennent la langue basque, mascarades et pastorales en Soule...) et une "fausse fête basque » folklorisée qui serait une sorte de « contrefaçon culturelle ». Contrefaçon culturelle qualifiée comme telle par certains Basques. La distinction entre ces deux univers ne serait-elle pas dès lors comprise dans un « capital » linguistique qui serait à lui seul capable d'effectuer la distinction et de marquer la ligne de démarcation ?

Afin de ne pas tout mélanger et de spécifier ses analyses, l'anthropologue aura tout intérêt à clarifier son étude en faisant appel à des dénominations distinctes qui pourront prendre place dans deux ensembles distincts. Dans celui de la « fête basque au Pays Basque » viendrait s'intercaler de nouvelles spécifications qui s'intègreraient à cet ensemble grâce à un sous groupe dénommé « fête des Basques bascophones au Pays Basque ». Du plus global au plus local, la focale du chercheur parviendrait à délimiter un ensemble humain constitué par une communauté voire une collectivité. Collectivité productrice de fêtes. Fêtes productrices de discours pouvant être analysés afin de permettre de spécifier la problématique de la recherche.

Dans son repérage des fêtes basques au Pays Basque le chercheur aura le choix dans l'élection d'objets d'études. Il pourra par exemple s'intéresser à la journée Herri Urrats qui se tient chaque année à Saint-Pée-sur-Nivelle autour du lac le premier samedi du mois de mai. Journée organisée en faveur des écoles en langue basque Ikastola par l'association Seaska. Là, grâce à une observation participante il pourra décrire la fête, étudier son programme (concerts de rock basque, bertsulari, danses basques, chants basques, jeux, repas,...) et analyser les diverses revendications portées par les acteurs. S'il souhaite prolonger sa recherche, il pourra partir dans la province de Soule en été pour assister à l'organisation d'un pastorale et rechercher dans le discours des acteurs, des organisateurs et des participants les liens qui unissent ce théâtre populaire avec la culture et l'identité basque. S'il poursuit son séjour en hiver, l'anthropologue aura l'occasion de participer à des mascarades qui constituent une période privilégiée par une étude des discours que la société souletine tient sur elle-même. Enfin, s'il procède préalablement à un apprentissage suffisant de la langue basque, il pourra assister à des joutes d'improvisateurs lors de repas ou lors de championnats.

Toutes ces occasions seront pour le chercheur des moments privilégiés où il aura à saisir selon quelles modalités ces différentes fêtes incarnent et redéfinissent sans cesse l'être basque dans ce qu'il était, dans ce qu'il est et parfois même dans ce qu'il aimerait devenir. Car la fête est union, réunion d'un temps passé et révolu réactivé pour l'occasion. Tradition revisitée (danses, chants, costumes...), savoirs faire remis au goût du jour, culture en train de s'inventer. Mais au-delà, elle est discours sur le passé, le présent et l'avenir. Discours qui énoncent une histoire (pastorales) où une actualité sur des modes fixés par la tradition (mascarades, bertsularisme). Discours qui vise non seulement à dire, des vérités, mais à «faire faire ». C'est dans cette 
incitation du faire que la fête trouve sa valeur subversive.

A chaque fête ses chercheurs. Pour ceux qui souhaitent s'intéresser aux pastorales, les recherches de folkloristes (Humboldt, Chaho, Michel, Vinson, Webster), de linguistes (Oyharçabal), de spécialistes du théâtre (Etchecopar) ou d'anthropologues (de Larrinoa, Dicharry) permettent d'analyser de quelle manière chaque discipline est venue apporter sa pierre à l'édifice de la construction d'une connaissance. Pour ce qui est de la danse basque, le lecteur pourra se tourner vers les travaux de chercheurs qui au cours des deux derniers siècles se sont penchés sur la question (Alford, Arbelbide, Constantin, Dassance, Desplat, de Larrinoa, Fourquet, Gallop, Guilcher, Harruguet, Hérelle, Inchauspé, Irigoien, Itçaina, Lekumberri, Luku, Otharbure, Michel, Oyhamburu, Peillen, Poueigh, Urbeltz...). Xabier Itçaina est déjà revenu sur cette thématique dans un article intitulé « Ohidura dantzatuak eta ipar Euskal Herriko gizartea » ce qui explique que nous ne nous y attarderons pas. Pour ce qui est du carnaval, il ne serait que trop conseillé au lecteur de parcourir les travaux de Thierry Truffaut, de Kepa Fernandez de Larrinoa et de nos travaux sur les mascarades en Soule. Ce qui nous intéresse dans le cadre de cette conférence sur la fête des Basques au Pays Basque est de focaliser notre attention sur les relations intimes qu'entretiennent sur ce territoire la fête et la politique.

\section{Une instrumentalisation politique de la fête basque}

La fête est ce que les acteurs veulent bien qu'elle soit. Les acteurs peuvent profiter de ces moments de «liberté surveillée » pour exprimer des opinions, des revendications, des appartenances, une solidarité, des différences. Quand de jeunes abertzale décident comme ce fut le cas par exemple pendant les fêtes de Pampelune de 2003 d'afficher sur une grande banderole, Bietan jarrai / Gora E.T.A, elle se fait vitrine d'une contestation, affichage d'utopies en marche. La fête reste un moment privilégié pour saisir la réalité sociale et en particulier les discours revendicatifs des militants. Les panneaux des institutions du gouvernement de Navarre deviennent pendant les fêtes de Pampelune de 2003 le lieu d'expression des critiques. Lassociation Euskal Herrian Euskaraz (au Pays Basque en basque) tague de son e facilement repérable et exprime son désaccord avec la politique linguistique menée dans cette province. Des autocollants (Que se vayan! Alde Hemendik! Torturadores !) demandent le départ des forces de répression, Garde Civile, police nationale, CRS. La hache et le serpent qui symbolisent l'organisation séparatiste basque E.T.A fait aussi son apparition dans les rues à l'occasion des fêtes. Une banderole plastifiée avec deux visages de militants et un texte (enbor beretik sortuko dira besteak) s'expose dans la rue à côté d'autres slogans composés sur des affiches en papier demandant de continuer la lutte. Lorsque l'affiche se trouve décrochée, il y a toujours un jeune pour la replacer correctement afin que le sigle E.T.A se donne à voir au grand jour. L'affiche est placée de telle sorte que tous les gens qui descendent la rue puissent la regarder. Elles viennent aussi s'ajouter aux tags déjà mis en place au cours de l'année comme sur la place du quartier Txantrea à côté du supermarché. Le nom de l'organisation n'est plus apparent seul le sigle persiste entouré du slogan bietan jarrai qui signifie continuer avec les deux.

Pendant la fête, les espaces publics sont investis et les politiques ne s'y trompent pas. Quand au Pays Basque sud, après l'accession des socialistes au pouvoir, ils décident d'exiger des propriétaires de bars et des organisateurs de buvettes d'enlever les photographies des 
militants politiques incarcérés, c'est qu'ils souhaitent faire barrage à ces vitrines festives mais aussi hautement politiques. Dans ces enlèvements de photographies, c'est la réalité du conflit politique qui ressurgit par temps de fêtes. C'est une liberté d'expression jusqu'alors tolérée qui est muselée par les forces de l'ordre. La liberté festive est surveillée, encadrée en fonction des idéologies en place. D'extraordinaire, la fête est rappelée à l'ordre par l'ordinaire et son cortège de lois, par sa logique de répression. La fête est teintée d'idéologique et porteuse de sens. Elle est grille de lecture qui permet de décoder une réalité. C'est le cas pour les pastorales qui interprètent l'Histoire, pour les mascarades qui reviennent sur l'actualité de la province de Soule et pour l'improvisation versifiée dans l'instant qui via ses improvisateurs dispensent ses vérités. Dans une société où une partie de la classe politique est privée de représentation, cette pratique culturelle est investie, ou plutôt devrions-nous dire surinvestie, par une surcharge de politique. Politique qui ne trouve pas de voie d'issue dans son domaine originel et qui pour se faire audible empreinte des voies périphériques, dérivées, culturelles. La culture est donc au Pays Basque plus qu'ailleurs chargée d'un poids politique qui devrait comme dans toutes véritables démocratie être porté par le politique.

Dans ces conditions, la frontière entre le politique et la fête est bien souvent poreuse. Les fêtes peuvent devenir un prétexte pour l'organisation de manifestations qui affichent des revendications et des appartenances politiques et idéologiques. L'exemple du groupe de jeunes qui mettent en avant pour les fêtes de Bayonne de 2011 une proposition alternative est là pour le rappeler. En effet, le groupe de jeunes militants a intégré dans son agenda alternatif des fêtes un jour des jeunes « gazte eguna » à la place Patxa dans le petit Bayonne et une manifestation prévue le jeudi 28 juillet. Il a de plus planifié une "mobilisation » le dimanche 31 à 11 heures trente en face de l'Eglise Saint André avec comme slogan : UPN tortionnaires, «UPN torturatzaileak! »

Nous le comprenons bien avec ces exemples. Il existe bien un monde entre les acteurs d'une même fête, entre un militant basque qui va manifester une réalité qui existe au Pays Basque à savoir la torture et un autre jeune qui va aller danser une chorégraphie lors d'une flash mob habillé en rouge et blanc sur une musique de Lady Gaga. Chacun va mettre dans la fête une part de ce qu'il est. La complexité de la fête découle du fait que l'ensemble de ces acteurs vont se retrouver au même moment et dans une même ville pour inventer une fête qui va renvoyer à des actes et des discours qui nous renseignent sur ce qu'ils sont.

Fête, bertsularisme et politique

L'art de bertsulari consiste à osciller en permanence entre vérité pure et vérité « manipulée ». Limprovisateur peut partir pour créer ses bertsus d'un fait réel qu'il va traiter de manière à faire rire. Il s'agira donc d'une plaisanterie même si celle si contient une part de vérité. On retrouve dans l'improvisation orale basque les trois catégories, les trois faits pointés par Jeanine Fribourg pour les « dichos» de la fête du Saint Patron en Aragon.

"Ceux qui, sérieux, ne supportent qu'une pointe d'humour, ceux qui sont drôles en euxmêmes, et enfin ceux qui, partant d'un évènement réel mais insignifiant, sont quasiment réinventés et interprétés sur le mode comique. » (Fribourg, 1996, p 150) 
Les différences qui concernent ces deux littératures orales sont manifestes au niveau de l'investissement de la charge comique des sujets. Alors qu'en Aragon, la politique est un thème qui n'admet que peu la plaisanterie, il est au contraire au Pays Basque un sujet qui est un thème traditionnel du rire et les improvisateurs n'ont de cesse de brocarder partis et hommes politiques. La politique est non seulement un thème qui admet au Pays Basque la plaisanterie mais c'est un sujet qui procure au public les meilleures occasions d'en rire. Par temps de crispations, se moquer des adversaires politiques, par exemple d'un Président de la communauté autonome basque socialiste espagnol, du PP, du PSOE mais aussi de l'UMP, du PS, du modem ou du FN est ici un moyen d'évacuer la pression accumulée par le conflit politique qui pèse sur les bertsularis et sur les auditeurs des joutes improvisées à des degrés divers. Par temps d'interdiction de partis politiques (ANV, Batasuna au Pays Basque sud), d'organisations de jeunes (Segi...) et par temps de répression de la gauche radicale nationaliste où les arrestations sont quasiment quotidiennes, la littérature orale est un moyen de contestation et de dénonciation de la réalité politique. Le rire est surinvesti d'une fonction perlocutoire et cherche à produire un effet sur l'allocutaire, à le faire passer à l'action par un acte de langage.

Il existe une continuité entre un t-shirt comportant la mention «Independentzia » porté par un bertsulari, une affiche demandant le retour des prisonniers politiques basques au Pays Basque incarcérés et disséminés dans les geôles françaises et espagnoles et les textes de l'improvisation orale. Vêtements, slogans, affiches et discours improvisés renvoient à une terminologie commune. Le discours intra-bertsularistique rejoint l'extra-bertsularistique. Lorsqu'en décembre 2008 plusieurs improvisateurs dont Andoni Egaña, Amets Arzalluz, Imanol Lazkano, Sustrai Colina, Sebastian Lizaso, Xabier Silbeira, Unai Iturriaga, Harkaitz Estiballes, Fredi Paia, Igor Elortza, Jon Maia, Xabier Amuriza, Jon Lopategi, Jon Enbeita eta Onintza Enbeita signent le manifeste pour demander la fin des condamnations à vie, synonymes de condamnations à mort pour les prisonniers politiques basques et la libération de ceux dont l'état de santé alarmant l'exige, leurs revendications rejoignent celles qu'ils expriment, de manière plus ou moins explicite, dans leurs énoncés des joutes improvisées. Officialisation de la langue basque, réunion des sept provinces sous une même entité, fin de la répression par les forces de l'ordre des deux Etats, français et espagnol telles sont les réclamations communes aux discours qu'ils soient internes ou externes au bertsularisme. Par temps de crise, les énoncés et les discours réactivent une actualité brûlante et rappellent que les bertsularis vivent parfois intimement le conflit basque, soit pour avoir des amis emprisonnés, soit pour avoir eux-mêmes été l'objet d'interpellations de la part des forces de répression (gendarmerie et police nationale française, guardia civil et police nationale espagnole). Par temps de crise, comme l'écrit le bertsulari Fredi Paya, les championnats se transforment en « cérémonie politique ». « (...) Txapelketak ekitaldi-politiko bilakatu zirela. » (Paya, Gazteiz, 2011, hitzaldia, Bertsolamintza)

Ridiculiser l'Autre, l'étranger qui peut prendre ici diverses figures comme celle d'un géomètre ou d'un juge c'est aussi à l'inverse s'affirmer soi-même, solidariser un groupe, une communauté par le biais d'autres figures antinomiques des précédentes comme par exemple, par celle du berger. Par le rire, les Basques attestent de leur vitalité et de leur identité. En ce sens le rire définit ce qu'ils sont et donne à voir leur « être » pour reprendre la formulation de Jean Haritschelhar. Se rassembler autour de l'improvisation orale c'est pour les Basques affirmer cette vitalité de l'être basque. Comme le notait Sustrai Colina dans un article paru dans 
le journal en langue basque Berria, la réunion autour du bertsu dépasse l'objectif strictement culturel. Lorganisation d'un championnat de bertsu au Pays Basque nord a un but similaire à celui que peut avoir Herri Urrats, la fête annuelle organisée par l'association Seaska pour soutenir les écoles basques. C'est donner aux gens l'occasion de se rassembler, de se réunir, de se regrouper pour affirmer tous ensemble l'attachement d'un peuple à son pays, à sa langue, à sa culture, à son identité. «Badu herrigintzatik».

Les cibles de l'improvisation orale basque ne manquent pas, de l'homme ou de la femme politique aux partis politiques, du patron et par extension du patronat à la presse, des curés et hommes d'Eglise aux financiers des banques, des Basques aux étrangers, des habitants de telle ou telle province à ceux de telle ou telle ville, des femmes aux hommes, des jeunes aux vieux, peu nombreux sont ceux qui échappent à la critique caustique, satirique et acerbe du bertsularisme. Seuls les prisonniers politiques basques ou les morts interdisent d'en rire. La souffrance de l'auditoire trace les frontières de ce que nous avons défini comme étant les limites du risible.

Le bertsulari est investi d'une autorité en tant que porte parole. Il a en ce sens une responsabilité et un rôle politique. Une sorte de sagesse qui ne peut s'exprimer dans le discours que si elle s'exprime aussi dans la pratique. Le bertsularisme passe par un apprentissage pratique. Ce n'est pas un apprentissage abstrait. C'est une pratique qui s'apprend par la pratique:

" une science pratique, un art que la pratique revivifie sans cesse, auquel l'existence lance sans cesse des défis. C'est ce qui fait que l'héritage ne survit qu'en changeant sans cesse : la transmission remodèle continuellement l'héritage en l'actualisant » (Mammeri, 1978 ).

Il a tout, comme l'amusnaw berbère :

" la capacité de dire au groupe ce qu’il est selon la tradition qu'il s'est donnée, par une sorte de définition par construction de concept qui lui dit à la fois ce qu’il est et ce qu'il a à être pour être vraiment lui-même. » (Bourdieu, 1978)

C'est dans la double faculté d'élasticisation de la réalité sociale et de réajustement à cette même réalité que la pratique tire sa vertu cardinale. Les Basques font de cette improvisation versifiée dans l'instant un espace de réaffirmation des liens identitaires et linguistiques en se référant en permanence à une culture locale, proclamée dans le discours, comme homogène. Cette pratique, invention et libération imaginale sans cesse revivifiée, est une réponse alternative donnée à une acculturation qui va crescendo, à une débasquisation de la population locale (spécifiquement au Pays Basque nord), à une World culture, à une globalisation, à une mondialisation, à une culture monde, à un village planétaire, qui «masquent l'horizon plus qu'elles ne le dévoilent» (Perrot, 2005). Le bertsularisme éclaire le passé, permet de penser le présent et de se projeter dans l'avenir. Il est trait d'union de l'ici au maintenant, du hic au nunc, reliance du temps avec l'espace. Il permet aux Basques, qui lui transfèrent une forte charge symbolique, de se dire, dans une alternance de rire et de sérieux, et de s'affirmer face à un monde en changement perpétuel accéléré. Il est celui par qui ils définissent et consolident 
leurs racines.

Les poètes basques sont des professionnels de la manipulation du verbe, de la société, du monde social. Ils présentent le monde social tel qu'ils voudraient qu'il soit. Leur discours est empreint de cette représentation et de cette volonté d'être dans un autre monde. Comme les Canaques, les Berbères ou toutes les sociétés en difficulté d'exister, les poètes du verbe basque sont placés dans une situation critique où leur identité collective est en crise, ce qui les porte à élaborer des projections plus ou moins fantasmatiques. La société basque telle que là rêve les bertsularis fait penser à ce que Feuerbach a dit à propos de Dieu : de même qu'on donne à Dieu tout ce qui nous manque, de même on donne à la société basque future et fantasmée tout ce que n'a pas la société basque d'aujourd'hui, tout ce qui lui manque et en premier lieu son indépendance. Dans cette reconstruction fantasmatique, l'ethnologie tout comme l'est le bertsularisme, peut être utilisée comme instrument idéologique d'idéalisation. Dès lors que quelques poètes basques pensent et disent qu'il pourrait y avoir un jour un Pays Basque indépendant il n'est pas impossible qu'il y ait un jour un Etat basque car nommer n'est-ce pas déjà faire exister?

Il convient de mentionner pour conclure cet article qu'au terme de cette réflexion sur la Fête au pluriel telle que nous l'avons définie de nombreuses questions mériteraient d'être approfondies. Elles pourraient interroger 1) les raisons qui expliquent la disparition et/ou la reprise de fêtes au cours du temps, 2) les transformations qui affectent ses fonctions (pacification sociale, contrôle social, transgression des normes, régénératrice, contestataire...) 3) les relations qu'entretiennent les collectivités locales et la fête. Collectivités qui mettent en avant le côté consommatoire de la fête et la pousse à devenir un simple produit de consommation 4) les modalités des processus d'« ethnicisation » qui les affectent et l'importance relative aux revendications et aux détournements de l'ordre social.

\section{Bibliographie}

ABAIGAR MARTICORENA, Federico, Irungo Euskal Jira, 1993.

ALTHABE, Gérard, et SELIM, Monique, Démarches ethnologiques au présent, Paris, LHarmattan (coll. « Anthropologie critique »), 1998.

BERTIN, Georges, «Interculturel et mondialisation: niveaux de communication culturelle, temporalité et sociétés », Esprit critique, vol.04 no.10, Octobre 2002, consulté sur Internet: http://www.espritcritique.fr

BERTIN, Georges, L'Imaginaire de la Fête locale, thèse de doctorat, Université de Paris 8 Vincennes à Saint Denis, 1999.

BIDEGAIN, Eneko, Patxa. Besta bai borroka ere bai, Gatuzain, 2010.

BOURDIEU, Pierre, «Entretien avec Mouloud Mammeri : Dialogue sur la poésie orale en Kabylie », Actes de la recherche en sciences sociales, n² 23, pp. 51-66, 1978.

CAILLOIS, Roger, Les jeux et les hommes, collection Folio, Gallimard, 1991.

CARO BAROJA, Julio, El estío festivo. Fiestas populares de verano, Madrid, Taurus, 1984.

CASTORIADIS, Cornelius, L'Institution Imaginaire de la société, Le Seuil, 1975.

CERTEAU, M. de, La Culture au pluriel, Paris, Christian Bourgois (1 ${ }^{\text {re }}$ éd. 1974),1980.

CUCHE, Denys, La notion de culture dans les sciences sociales, Paris, Editions La Découverte, 1996. 
Le Débat, Culture et Politique, n70, mai-août 1992.

DICHARRY, Eric, Du rite au rire. Le discours des mascarades souletines. Thèse de doctorat, Ecole des Hautes Etudes en Sciences Sociales, Paris, 2009. http://www.euskonews.com/0517zbk/ gaia51701fr.html

DICHARRY, Eric, Anthropologie de l'art contemporain basque au XXIème siècle. Conférence donnée dans le cadre des conférences d'Eusko Ikaskuntza à la médiathèque de Biarritz en mars 2009. http://www.euskonews.com/0490zbk/gaia49001fr.html

DICHARRY, Eric, "De l'expérience du terrain aux théories ethnolinguistiques ", Congrès de Basauri (Euskal Herrian folklore eta etnografia ikerketa : atzo, gaur, bihar) organisé par la société d'études basques, Eusko Ikaskuntza, Lankidetza 51, 2008.

DICHARRY, Eric, «Approche ethnolinguistique des mascarades souletines », Oihenart, n¹6, pp. 87-136, Bayonne, 1999.

DOUGLASS, William A., LYMAN, Stanford M., « Ethnicity: strategies of collective and individual impression management », Social Research, 15, 1973, p. 344-365.

DUVIGNAUD, Jean, Le don du rien. Essai d'anthropologie de la fête, Stock 1977.

DUVIGNAUD, Jean, Fêtes et civilisations, Scarabée, 1984.

FABRE, Daniel, Carnaval ou la fête à l'envers, collection Découverte, Gallimard, 1992.

FRIBOURG, Jeanine, Fête et littérature orale en Aragon, LHarmattan, 1996.

GARAT, Isabelle, Fêtes en ville, villes en fête, Flesselles, 2000. http://www.cafe geo.net/article. php3?id_article=443

GARAT, Isabelle, La recomposition des espaces sociaux dans une ville moyenne: l'exemple de Bayonne, thèse de géographie sous la direction de G. Di Méo, Université de Pau et des Pays de l'Adour, 1994, 405 pages.

ITZAINA, Xabier, « La Fête-Dieu face à ses interprétations en Pays Basque nord », in http:// www.euskonews.com/0102zbk/gaia10205fr.html.

ITZAINA, Xabier, «Danse, rituels et identité en Pays-Basque français », Ethnologie française, 26, 3, 1996, p. 490-503, eta « Dantza Ipar Euskal Herrian : herri kulturatik euskal kulturarat », XV Jornadas de folclore y cultura tradicional, Ortzadar, Iruña-Pamplona, Sukil, octobre 2000.

LABORDE, Denis, « Politique culturelle et langue basque. Le Centre culturel du Pays Basque (1984-1988)», Lapurdum, 2, 1997, p. 339-354.

MAFFESOLI, Michel, L'ombre de Dionysos : contribution à une sociologie de l'orgie, Paris, librairie des Méridiens, 1985.

PERROT, Martyne, « Quand faire sien, c'est faire autrement », in Communications, Volume 77, pp. 5-16, Année 2005.

ROY, Claude, Paul Veyne et René Char au passage, Gallimard, « NRF », 1997.

TODOROV, Tzvetan, Nous et les autres. Réflexion française sur la diversité humaine, Éditions du Seuil, Paris, 1989.

URFALINO, Philippe, Linvention de la politique culturelle, Comité d'Histoire du Ministère de la Culture, Paris, La documentation Française, 1996.

VEYNE, Paul, L'Inventaire des différences. Leçon inaugurale au Collège de France, Paris, Éditions du Seuil, 1976.

VEYNE, Paul, Comment on écrit l'histoire, Paris, Seuil, 1971.

WARNIER, Jean-Pierre, La mondialisation de la culture, Paris, La découverte, 1999. 